\title{
Désolation, vocation, exemplarité : les lettres de consolation de Théodore de Beringhen (1686-1700)
}

\section{Chrystel Bernat}

\section{(2) OpenEdition}

\section{Journals}

Édition électronique

URL : http://journals.openedition.org/rhetorique/532

DOI : $10.4000 /$ rhetorique.532

ISSN : 2270-6909

Éditeur

UGA Éditions/Université Grenoble Alpes

\section{Édition imprimée}

ISBN : 978-2-37747-010-5

\section{Référence électronique}

Chrystel Bernat, «Désolation, vocation, exemplarité : les lettres de consolation de Théodore de Beringhen (1686-1700) », Exercices de rhétorique [En ligne], 9 | 2017, mis en ligne le 21 juin 2017, consulté le 12 septembre 2020. URL : http://journals.openedition.org/rhetorique/532 ; DOI : https:// doi.org/10.4000/rhetorique.532

Ce document a été généré automatiquement le 12 septembre 2020.

\section{(c)}

Les contenus de la revue Exercices de rhétorique sont mis à disposition selon les termes de la Licence Creative Commons Attribution - Pas d'Utilisation Commerciale - Partage dans les Mêmes Conditions 4.0 International. 


\title{
Désolation, vocation, exemplarité : les lettres de consolation de Théodore de Beringhen (1686-1700)
}

\author{
Chrystel Bernat
}

1 Une première étude sur le langage de l'émotion dans la littérature de résistance huguenote de la période révocatoire et celle de consolation du dernier tiers du XVII ${ }^{e}$ siècle témoigne d'un propos saturé de références scripturaires ${ }^{1}$, trait spécifique d'un discours protestant alors fondé sur une lecture biblique des temps. Ce discours relève en partie de la langue (ou patois) de Canaan, qui désigne l'intrication des données scripturaires au texte de l'auteur, et que j'ai associé ailleurs à une théologie subliminale qui, en entrelaçant le temps présent au temps biblique, s'apparente à un moteur langagier et idéel à trois temps : celui-ci délivre un message premier jouant d'allusions à l'appui d'une symbolique implicite qui en favorisant l'identification lève une perspective et, avec elle, une attitude à suivre en dispensant d'avoir à la formuler dans sa radicalité . En recourant à une rhétorique biblique, ce dialecte joue d'insinuations et de filiations tacites par le biais desquelles s'énonce un discours de militance puisant en particulier dans l'histoire accidentée de l'Israël biblique, tantôt exhortatif et menaçant, tantôt consolatoire et compatissant qui, volontiers critique et moralisant, n'est pas sans tonalité politique par la fidélité à la Réforme qu'il défend et l'insoumission qu'il suppose au programme de catholicisation du roi. Le discours proprement consolatoire n'échappe pas à ces divers registres de langue, pris à ce jeu de résonances bibliques et de modèles scripturaires, d'analogies et d'allégories établies au service de la défense d'une éthique de la foi réformée.

2 Ce tamis théologique est d'un attrait épistémologique fort pour qui veut saisir le double discours qui se loge dans le propos exhortatif et la rhétorique combative. Sans engager, en ce premier volet, l'examen du corpus des lettres proprement dit, il importe d'en souligner l'intérêt analytique pour l'appréhension du recueil. Nous verrons, ne seraitce que furtivement, l'effet de cet encodage biblique et l'utilité de son décryptage dans l'approche de la consolation elle-même. 
3 Après l'analyse des discours huguenots de censure, c'est dans l'optique d'un examen comparatif des discours de fraternité que s'est imposé ce recueil de lettres de consolation paru de manière anonyme, au Refuge, dans les toutes premières années du XVIII ${ }^{e}$ siècle sous le titre : Cinquante lettres d'exhortation et de consolation sur les souffrances de ces derniers tems \& sur quelques autres sujets; écrites à diverses personnes par Monsr. D. V. B. pendant ses exils \& ses prisons en France, \& depuis que par ordre du Roi, il s'est retiré en Hollande, La Haye, chez Jean Kitto, 1704. Son intérêt est multiple, d'une part dans la mesure où il est représentatif d'une partie du discours apologétique de la minorité confessionnelle huguenote du dernier tiers du XVII ${ }^{\mathrm{e}}$ siècle, d'autre part, parce qu'il a été peu sinon pas étudié - cet opuscule étant aujourd'hui encore vierge de toute analyse systématique ${ }^{3}$.

4 Le syntagme "désolation, vocation, exemplarité », qui explore le thème de la consolation aux prises avec l'affliction spirituelle, la revendication confessionnelle et la représentativité communautaire, vise ici à introduire aux fonctionnalités du discours de consolation, en interrogeant sa charge exhortative et proprement militante confrontée aux effets de l'oppression religieuse et à l'enjeu d'une proclamation identitaire bousculée par la politique louis-quatorzienne d'extirpation du protestantisme. Ce titre invite à l'examen conjoint de la typicité de la figure du consolateur/confesseur élaborée par Beringhen et de la codification du discours de consolation réformé qui, à cette heure du moins, est de l'ordre du témoignage et de l'engagement prescriptif, en d'autres termes, de l'exhortation combative.

5 Si l'on ne peut donc s'en tenir à l'idée d'un propos tout entier compassionnel, en quoi peut-on considérer que le discours de consolation spirituelle relève d'une forme de militance active? Quels en sont les sujets et les modalités discursives? Y a-t-il un lexique caractéristique? Comment entend-on consoler et quelles formes épouse la consolation, quelle intensité et variations la caractérisent? Au reste, qu'est-ce que consoler (plaindre, réconforter, assister, galvaniser) et qui console qui ? La consolation réside-t-elle dans la considération de la douleur ou dans le partage des afflictions, autrement dit relève-t-elle du domaine du soutien ou de l'expérience? Quelles figures véhicule-t-elle et, de manière transversale, que nous dit la figure spécifique du confesseur ${ }^{4}$ de la consolation elle-même, ou comment cette position adoptée par l'auteur nous renseigne-t-elle sur la particularité du discours de consolation huguenot ? En quoi celui-ci le modèle? Doit-on considérer qu'il y ait un dispositif consolatoire lié à la figure spécifique du confesseur? De quelles modalités religieuses de la consolation témoigne la littérature d'édification huguenote? Décèle-t-on une liturgie consolatoire (quelle place du silence, des pleurs, des lamentations, de la considération des fautes et de l'annonce de la grâce, de la prière, des gestes de compassion, de la déclamation de la foi)? Quels registres gouvernent le discours de consolation protestant de la période révocatoire et quel rôle les réformés lui assignent-ils?

6 Voici énoncées quelques-unes des interrogations soulevées par le sujet que cette première partie de l'enquête n'aborde cependant qu'à la marge, faisant le choix d'une présentation des pièces liminaires qui encadrent ce discours de consolation. Il s'agit ici d'évaluer les données matérielles dans l'optique de dégager quelques caractéristiques de la rhétorique consolatoire appréhendée à partir de son écrin éditorial. Commençons donc par une présentation détaillée de la source et de son auteur, de façon à considérer le cadre spécifique d'élaboration du discours de consolation spirituelle, et d'en retirer quelques particularités à la lueur des enjeux qui l'articulent et le structurent au 
tournant des $\mathrm{XVII}^{\mathrm{e}}$ et $\mathrm{XVIII}{ }^{\mathrm{e}}$ siècles tandis que les protestants se trouvent exposés à une catholicisation forcée.

7 Voyons en premier lieu la source, la composition de l'ouvrage, les éléments d'identification et d'attribution, en abordant le contenu des pièces du recueil.

\section{L'auteur, l'imprimeur - Correspondance d'un insoumis}

8 Paru de manière anonyme sous les initiales $D$. V. B., le recueil a été au XVIII siècle attribué par Antoine Court à Théodore de Beringhen, ancien conseiller au Parlement de Paris dont les parents se sont signalés par leur refus d'abjurer la foi protestante en dépit des disgrâces et des exils forcés (le père, secrétaire du roi et membre du consistoire de l'Église de Charenton, est retenu en février 1686 à Montargis, tandis que la mère, d'abord détenue en ce lieu avec son époux et l'une de leurs filles sous la surveillance des dragons, est enfermée ensuite à proximité de Paris au couvent de Gercy ${ }^{5}$ ). L'identification de l'auteur et l'attribution sont en cela conformes au nom que l'un des exemplaires de l'ouvrage porte au crayon : « Deodatus van Beringhen ${ }^{6}$ ».

9 En 1868, Jules Chavannes considère pourtant que ce recueil est attribuable à deux noms : Beringhen et de Vrigny, tous deux emprisonnés à la Bastille en 1686, tous deux confesseurs répondant au profil de l'auteur, émigrés aux Pays-Bas et compagnons du comité de Hollande travaillant depuis le Refuge au rétablissement des Églises réformées de France ${ }^{7}$. Un siècle plus tard, l'attribution semble néanmoins tranchée. En 1972, le répertoire de Pierre M. Conlon l'attribue définitivement à Théodore de Beringhen ${ }^{8}$.

Fils aîné d'une lignée de quinze enfants dont la plupart meurent en bas âge, Théodore de Béringhen est originaire d'une famille hollandaise dont plusieurs membres se sont signalés à la cour d'Henri IV et de Louis XIII ${ }^{9}$. Les frères Haag le présentent marié depuis janvier 1685 à Élisabeth Marie de Goyon (1661-1742), fille du baron de Marcé. À l'exception de son frère Frédéric, sieur de Langarzeau, cornette de cavalerie qui se convertit en 1686 après quelques mois passés dans les cachots de la Bastille, ses frères et sœurs seraient demeurés huguenots en dépit de l'oppression. Théodore passe pour avoir donné l'exemple, à l'inverse de son épouse qui se convertit à la suite de son enfermement au couvent des Filles du Saint Sacrement puis dans celui dit des Nouvelles catholiques ${ }^{10}$.

11 Huguenot fervent (né en 1644 et mort autour de $1703^{11}$ ), emprisonné d'abord à la Bastille à l'été 1686 (Lettre IV) puis au château de Loches (en Touraine) à partir de l'automne 1687 (Lettre XVI du 7 septembre 1687), Beringhen est expulsé de France et se réfugie (depuis La Rochelle où il fut transféré) aux Provinces-Unies, en premier lieu à Amsterdam d'où il écrit en mai 1688 (Lettre XXIII), puis à Rotterdam en février 1690 (Lettre Xxıx). Signant diverses lettres de «Hollande», Beringhen est membre (à partir de 1693, ou au plus tard en 1694, et encore en 1697) du comité des réfugiés de Hollande qui, avec Élie Benoist et Pierre Jurieu, de Vrigny, de Chenaille et de Peray, œuvre au soutien des calvinistes français et au rétablissement des Églises réformées de France dispersées par la politique religieuse oppressive de Louis XIV. C'est d'ailleurs chez Beringhen que le Comité se réunit pour la première fois le 18 mars $1694^{12}$. Son nom figure parmi les réfugiés que les exilés en Suisse mandatent depuis Berne, le 26 mai 1697, pour intervenir auprès des plénipotentiaires des princes protestants afin d'obtenir une amélioration du sort des réformés français à l'occasion des tractations de 
la paix de Ryswick avec le roi de France ${ }^{13}$. Le discours de consolation (et volontiers d'édification) publié en 1704 est donc celui d'un homme engagé dont les contemporains saluent " la constance à l'épreuve » et la piété ${ }^{14}$. Au reste, ce recueil ne serait pas la première publication de l'auteur: Beringhen mentionne un premier ouvrage, "un essay - écrit-il - [...] donné il y a quelques années", qui, selon lui, aurait été reçu favorablement, mais dont on ne connaît la teneur, n'en trouvant pas même trace ${ }^{15}$.

On peut s'interroger sur la raison d'une parution anonyme (à tout le moins semianonyme) dès lors que Beringhen, libéré sur ordre du roi et exilé aux Provinces-Unies, se trouve en sûreté au Refuge dès 1688 . On pourrait y voir la volonté de ne pas compromettre les chances de libération de ses proches parents (ou de ses connaissances) prisonniers du roi - une parution signée pouvant dissuader le souverain de les émanciper. Or à cette date, Béringhen se présente dégagé de toute nécessaire prudence. S'il est vraisemblable qu'il ait cherché, au moins un temps, à préserver son épouse, restée en France, de toutes formes de représailles (l'auteur fait part de " considérations domestiques qui engageoit a ne hazarder rien»), à l'heure où son recueil paraît, celle-ci, passée au catholicisme depuis 1686, est à l'abri de toute vexation, et lui-même se dit libéré de toute " considérations particuliéres ${ }^{16}$ ". À moins que l'hypothèse d'une publication posthume se confirme, et n'explique l'anonymat partiel (l'auteur n'ayant pas eu le temps de signer l'ouvrage ${ }^{17}$ ), il est malaisé de comprendre pourquoi ce recueil paraît sans signature.

La date de publication aussi intrigue, car en 1704, près de vingt ans se sont écoulés depuis l'acte révocatoire. Si l'oppression persiste avec plus ou moins de vigueur dans le royaume, le gros des persécutions est passé et rien a priori n'explique le choix d'une publication aussi tardive, que l'auteur pourtant tient désormais pour pressante : « $\mathrm{Si}$ j'ay eü des raisons d'en différer l'édition jusqu'à présent, j'en ai d'autres de ne la retarder pas plus long-temps». Sans s'ouvrir sur le motif de cette urgence, Beringhen s'explique sur le report de l'édition de l'ouvrage (auprès de « ceux qui [...] s'étonneront, peut-être, d'abord qu'on ait attendu si longtemps à le mettre au jour [...] après avoir laissé passer le temps de leur plus grande utilité ») par la volonté de ne pas exposer ses interlocuteurs et destinataires que ses lettres, publiées trop tôt, auraient désignés aux autorités. Reste à évaluer ce qui en détermine la publication impérieuse en 1704 et ce que recèle le partage de ce propos consolatoire alors même, précise-t-il, que « chacun a pris son parti en France » (Avertissement $\left.*_{5 v^{\circ}}\right)$. Si tout est ainsi joué, à quoi bon écrire et pourquoi cette précipitation soudaine, cette édition instante, a fortiori sur le mode consolatoire?

Publié à La Haye, le recueil paraît chez Jean Kitto, "marchand libraire dans le Spuystraat» précise la page de titre, désignant le quartier des libraires de la ville. Johannes Kitto figure dans le catalogue de la Bibliothèque royale des Pays-Bas en qualité d'imprimeur en activité à La Haye de 1700 à 1733 . Il ne s'agit donc pas d'un nom d'emprunt ni même d'un nom fantaisiste comme souvent au Refuge au temps de la Révocation ${ }^{18}$.

\section{Situation d'énonciation - Le confesseur. Consolation au miroir}

D'emblée, dès l'intitulé, dans l'Avertissement et la dédicace $\left({ }^{*} 2 \mathrm{v}^{\circ}\right)$, l'auteur se présente en qualité de confesseur dont témoignent ses exils et les peines de prison, ou pour mieux 
dire le consolateur épouse les traits du confesseur récemment délivré des fers, dont les écrits portent traces de l'adversité et d'une foi soumise à l'épreuve de l'oppression. Beringhen endosse la figure topique de l'affligé combatif, vainqueur du doute et des tribulations, dont la félicité réside dans la patience et la résolution à endurer l'épreuve ; il incarne la trajectoire de l'opprimé victorieux, dans laquelle l'humiliation des hommes préfigure la béatitude, sa profession constante de la vérité servant, en cette littérature apologétique, de modèle.

16 Il considère ses lettres comme les vestiges d'un temps d'affliction durant lequel Dieu a exercé sa fidélité, liant le discours de consolation à l'expérience de la foi, sur le mode de la défense d'une croyance : la consolation ne relève pas ici du domaine de la spéculation mais d'une piété active et d'un vécu partagé, qui postulent une proximité de souffrances (nous y reviendrons). L'intitulé même du recueil lie le propos exhortatif à l'acte de consolation. Au reste, la péricope placée en page de titre - « Israël est sainteté à l'Éternel, tous ceux qui le dévorent seront trouvez coupables, malheur en a[d]viendra. Jer. $2 »-$ porte la marque du combat que recouvre ici le discours consolatoire, en promettant la destitution des adversaires et en engageant les réformés à la persévérance : la consolation se trouve enchâssée dans l'annonce de la protection de Dieu malgré l'épreuve. Cette référence à Jr 2,3 est éloquente : d'une part du fait de son actualisation par l'auteur, qui use du présent tandis que le texte biblique insiste sur la protection passée et perdue qui nécessite d'être regagnée; d'autre part du fait de la récrimination implicite qui accompagne la consolation, car Jr 2, qui annonce l'élection d'Israël, dénonce simultanément sa trahison et la complainte de Dieu appelant son Peuple élu à revenir de son infidélité ; un discours à double détente pour les lecteurs, qui éclaire la teneur du propos consolatoire : à la fois réconfortant, comminatoire et prescriptif à l'égard des apostats, la bienveillance s'articulant à la plainte.

Ce discours a pour spécificité d'être le fait d'un auteur qui est partie prenante de l'affliction: Théodore de Beringhen est à la fois le consolé et le consolateur dans un processus consolatoire en miroir concave et convexe, destiné en se consolant à consoler ses compagnons d'infortune et à les édifier par l'exemple ${ }^{19}$. Son discours ambitionne de soutenir l'épreuve des autres par le témoignage de l'« expérience» personnelle (en l'occurrence l'expérience de « la vertu de [1]a Parole »). À l'opposé de la définition qui a pu en être proposée ${ }^{20}$, la consolation est ici moins de l'ordre de la compassion - d'une parole tierce, c'est-à-dire extérieure, sur la souffrance - que du partage intime des vicissitudes. Elle n'est pas une simple façon de prendre part à l'affliction, mais procède d'une communion d'épreuves ${ }^{21}$, et semble trouver sa justification dans la proximité des souffrances desquelles Beringhen entend valoriser l'endurance et ce qui lui a permis de ne pas céder à l'oppression, de ne pas se résigner à l'apostasie. Elle n'a pas pour finalité de convenir d'un désespoir (sinon pour mieux soutenir une foi proscrite dont le maintien de la persécution témoigne paradoxalement de la justesse), mais d'établir une parenté à partir de laquelle se légitime une admonestation, s'énonce un encouragement, se prescrit une recommandation. La consolation est à la fois l'espace d'une contiguïté et le ressort actif d'une parole programmatique (de foi, de vertu, de fidélité). Le siège d'une union qui toujours se projette par-delà la douleur qui la motive. Dit autrement, la consolation (rivée, chez l'auteur, dans l'amour de Dieu) réside au-delà de son motif premier (la persécution religieuse). La consolation fait l'effet d'une double circularité, d'une part entre l'objet de la douleur et sa résolution, d'autre part dans le 
partage des épreuves : Beringhen entend consoler en partageant les motifs de sa propre résistance à l'oppression catholique.

\section{Le livre - Un écrin poly-stylistique} couvrant 470 pages du volume (précédées de 19 pages signées mais non paginées) assorties (dans l'ordre) d'une Invitation chrétienne (lapidaire mais fort instructive), d'une dédicace A leurs Hautes puissances, les seigneurs des États généraux des Provinces-Unies des Païs-Bas (d'une longueur de 6 pages signées *2 à *4), d'un Avertissement au lecteur (de 8 pages signées seulement ${ }^{*}$ ), d'une Prière (de 4 pages signées**). Suit la publication numérotée des Lettres I à L (p. 1 à 470) après lesquelles figurent un court Article d'intercession intitulé Dans mes prières, depuis la révolte de ma femme (non signé, sans date ni emplacement chronologique manifeste), puis un Indice des lettres (non signé) et un erratum (Fautes à corriger) sur lequel s'achève l'ouvrage ${ }^{23}$. Reprenons en détail ces rubriques comme autant de pièces introductives et additives utiles à l'approche du discours de consolation - l'examen de certaines d'entre elles étant incorporé à diverses analyses.

L'Invitation chrétienne se présente sous forme de dédicace universelle aux affligés, adressée «A tous ceux, qui, en quelque lieu que ce soit, invoquent le nom de Jésus Christ ; Seigneur d'eux \& de moi ». D'emblée, ce discours de consolation se trouve placé dans le registre de la foi, et entend jouer d'une fraternité spirituelle, exprimée à l'attention des membres d'une communauté qui se présente moins sous son aspect confessionnel (protestant, huguenot) que dans sa revendication proprement chrétienne. C'est là une donnée identitaire que l'on peut lire de manière contradictoire, dans la mesure où l'absence apparente de polémique (il n'y a là aucune formule confessionnalisante ou séparatiste stricte) se trouve contrebalancée par l'identification aux partisans du Christ: sans se réclamer protestant, on se définit néanmoins vrai chrétien. À ce titre, l'invitation n'est pas sans évoquer la ligne de rupture réformatrice qui veut que les adversaires catholiques invoquent le pape quand les protestants s'en remettent directement à Dieu, car c'est précisément à partir de ce type de formule qu'ils s'en distinguent alors volontiers depuis le XVI ${ }^{\mathrm{e}}$ siècle. Dédier l'opuscule «à tous ceux qui [...] invoquent le nom de Jésus » n'est pas sans dissocier le camp des partisans du Christ de ceux qui, de manière sous-entendue, ne le sont pas. Contrairement donc à ce qu'une première lecture peut laisser accroire, la formule n'est pas sans revendication confessionnelle sous-jacente ni marqueur identitaire fort. Sans bannière polémique manifeste, il pourrait y avoir là un renversement de la critique d'hérésie, qui, d'un mot d'un seul, départage les adversaires et bascule dans le camp des oppresseurs catholiques. Ceci dit, l'effet se trouve atténué par le lieu de l'édition, en l'occurrence La Haye, haut lieu d'exil huguenot. En pays de Refuge tout entier protestant, l'effet minoritaire s'émoussant, a-t-on besoin de se présenter autrement que comme chrétien? Il importe donc de relativiser ce ressort controversiste. Il n'en reste pas moins un étendard sous lequel s'affiche un particularisme doctrinal suffisamment éloquent, selon l'auteur, pour que les destinataires y reconnaissent une appartenance : adressée à une internationale chrétienne ("à tous ceux qui, en quelque lieu que ce soit, invoquent le nom de Jésus (hrist»), la dédicace veut faire entendre (sinon prétend à) l'universalité d'une complainte : le discours de consolation se joue ici 
des frontières, postule un langage de foi unique. Peu ou prou polémique, l'auteur s'adresse à une communauté de persécutés endurants à l'attention desquels il entend partager ses méditations (et ses encouragements) sur les « souffrances de ces derniers tems ", tel que l'indique le titre de l'ouvrage. Il y a aussi une volonté de s'associer au Christ que l'auteur place sur le registre de l'union (« Seigneur d'eux \& de moi »).

L'Invitation se poursuit par une péricope tirée du Psaume 66: «Vous tous qui craignez [i.e aimez] Dieu, Venez, écoutez; \& je raconteray ce qu'il a fait à mon âme ». C'est là un indice de la dimension prescriptive et interprétative du discours de consolation qui consiste à donner un sens à l'affliction, qui allègue un témoignage, prétend à la mise en partage d'un vécu sur le mode de l'exemplarité et de l'appel (d'une proximité réflexive), qui place de fait la consolation dans une dynamique motrice, loin de la passivité que la seule empathie lui confère. Dans le choix de cette péricope, il y a déjà aussi l'annonce d'une promesse. Fonder l'invitation sur le Psaume 66 - qui est un psaume de louange au Dieu libérateur (qui éprouve ses enfants mais ne laisse pas vaciller les endurants et qui offre l'abondance aux persévérants Ps 66,8-12) - renseigne sur la tonalité exhortative fondamentalement libératoire du discours de consolation de Beringhen, qui est ici de l'ordre de l'émancipation de la contrainte et de l'exhortation à la fidélité spirituelle. Une fidélité placée sous le signe d'une patience confiante, dont l'assurance de la victoire réside dans la constance. Le programme est déjà annoncé. On y trouve la quintessence du discours de consolation, qui sert à considérer les fruits d'une fidélité tenace à l'heure de la tourmente, à défendre l'idée selon laquelle la félicité procède des tribulations et le trouble recèle des promesses. La consolation est du domaine de l'édification (à moins que ce ne soit l'inverse, nous le verrons) et a ici valeur d'instruction religieuse ${ }^{24}$.

21 La dédicace $\left({ }^{*} 2-{ }^{*} 4\right)$ à Leurs Hautes puissances, les seigneurs des États généraux des ProvincesUnies des Païs-Bas, non datée mais vraisemblablement rédigée peu avant l'édition du recueil (en 1704), est à la fois le témoignage d'une allégeance vis-à-vis des protecteurs, et une façon de placer l'ouvrage, et avec lui le propos consolatoire, sous l'autorité des puissants, le haut patronage des seigneurs protestants du Refuge. La dédicace est adressée au corps souverain suprême des institutions républicaines des ProvincesUnies qui concentrait les pouvoirs législatifs et se trouvait notamment en charge de la politique religieuse ${ }^{25}$. Faut-il y lire un processus de légitimation? Sans doute s'agit-il moins d'une accréditation que de la considération du soutien des États généraux - des "libéralités ${ }^{26}$ " accordées aux exilés - dont bénéficient en bout de chaîne les réformés français les plus endurants. En revanche, le fait d'accéder aux requêtes des huguenots (" chassez de leur patrie»), que l'auteur souligne avec gratitude, dissimule peut-être une aide des Hautes puissances à l'édition même du recueil, qui fait de la littérature d'édification une préoccupation partagée par les instances du Refuge, et du propos consolatoire individuel un discours de soutien communautaire.

La dédicace permet également d'accéder à diverses considérations de l'auteur qui y présente son ouvrage comme un "petit recueil de lettres [...] écrites autrefois [...] au milieu de l'épreuve » afin de consoler et de soutenir les espérances des « enfans de Dieu dans leurs épreuves" $\left({ }^{*} 2 \mathrm{v}\right)$. La dimension temporelle (l'« autrefois ») suggère un certain recul. La date de rédaction des lettres, antérieure à l'élaboration a posteriori du volume tiré de sa correspondance, soulève la question d'une possible réécriture. Malgré la datation ancienne des missives, la dédicace suggère des retouches, l'auteur stipulant réunir des «lettres [...] écrites autrefois pour la plus-part», non pour la totalité : la 
formule laisse envisager de possibles adaptations. Le discours de consolation relève donc d'un processus d'écriture modulable, potentiellement superposable, ici articulé à un genre hybride que renforce la connaissance théologique de l'auteur. Chez Beringhen, il épouse la double forme de la correspondance spontanée et d'une homilétique épistolaire bardée de références scripturaires.

Ce qui est sûr, c'est que ces missives ne sont pas lues ni le discours de consolation entendu pour la première fois lors de leur édition en recueil : adressées à une foule de destinataires au profil disparates et aux épreuves chaque fois distinctes (là peut-être se repère un tri de l'auteur), ces lettres ont déjà circulé à la date de leur parution (l'auteur confie - ${ }^{*} 2 \mathrm{v}^{\circ}$ - être parvenu à les envoyer et à atteindre leur destinataire respectif malgré les aléas de l'emprisonnement et de l'exil). Nous ne sommes donc pas face à un discours de consolation simplement segmenté, et débité en missives choisies, mais face à un recueil de lettres écrites au gré des épreuves conjoncturelles dont il importera d'évaluer le déploiement des sujets d'édification, la variété des objectifs et l'évolution des dispositifs consolatoires sur près de quinze ans ${ }^{27}$.

L'Avertissement au lecteur, assez fourni (8 p., signées uniquement ${ }^{*}$ ), est riche d'enseignements. L'auteur y expose les raisons de la publication de ses lettres. Retenons pour l'instant que récusant toute prétention littéraire, il écrit vouloir simplement partager les «sentimens d'un cœur touché par ce qu'il exprime », sans " tour ingénieux des pensées ». Précisant les conditions de rédaction, Beringhen y présente ses procédés d'écriture, forgeant son discours de consolation dans une immédiateté adossée à l'Écriture : il postule un discours de consolation sans apprêt ni manières affectées dont la force procède de la simplicité ( "J'écrivois les choses naturellement comme elles me venoient dans la pensée, \& un passage de l'Ecriture qui se présentoit a propos pour m'éclairer ou pour me fortifier, dans l'état où je me trouvois»). La justesse de la consolation a à voir avec la spontanéité de l'adresse. Elle ne se veut pas une élaboration mais une déclaration libre sinon inspirée - les "affectations d'un style plus médité " menaçant, selon l'auteur, d'obscurcir la conscience. Le propos consolatoire, qui se présente comme l'expression «de simples idées de la Vérité sans fard \& sans ornement ", est empreint d'une dimension parénétique: la consolation relève d'un discours qui se prétend fondé sur la Vérité, et réside pour l'auteur dans une authenticité de foi qui offre de trouver la force de fidélité nécessaire à une croyance ébranlée par les vicissitudes. Chez Beringhen, la consolation est le produit d'un organe duquel procède l'efficacité : le produit d'un cœur à cœur qui lie la source et l'effet de la consolation ( $«$ Je donne mes Lettres, telles que le cœur me les a dictées, telles qu'elles m'ont consolé quand je les ai écrites, \& qu'elles ont touché ceux qui les ont receuës »). Exempte de toute forme d'écran, la consolation s'apparente à une eucharistie des cœurs. Ce qui n'exclut pas une tension entre le caractère a priori primesautier des lettres et la dimension méditative que l'auteur leur reconnaît un peu plus loin.

Exprimant les vœux du consolateur, la Prière (signée ${ }^{* *}$ ) éclaire le ressort principiel du discours de consolation de Beringhen qui répond du devoir de glorification de Dieu après la délivrance des afflictions. Ce qui indique ici que la consolation ne répond pas au seul besoin de l'affligé mais du consolateur lui-même, bénéficiaire d'une sagesse dont il se doit désormais de rendre compte à la gloire de Dieu, non de ses propres forces («ce sont tes seules gratuitez qui m'ont préservé»). Consoler revient dans cette littérature apologétique à louer la miséricorde divine. La consolation relève dans l'apologétique réformée d'un devoir: celui d'ouvrir la bouche, «en cantiques et en 
loüanges ", pour confesser sa reconnaissance et annoncer l'abondance des consolations qui suit l'épreuve et la détresse des coreligionnaires. Priant que lui soit donné à entendre la gloire que Dieu a révélée dans sa propre délivrance - là réside l'essentiel du motif et du message consolatoires -, l'auteur fait de la consolation une chaîne d'amour : «Tu m'as donné de triompher de la Bête [...]. Fay que dans les amertumes de ma vie ; \& ma condition présente, je regarde incessamment à la gloire que tu m'as révélée [...]. Que [...] je puisse, de la même consolation dont tu m'as consolé, consoler aussi les autres dans leurs différentes afflictions ». Au reste, Beringhen déclare n'écrire "que pour les cœurs capables de l'amour de Dieu ", sans en exclure pour autant les parjures et les timides, qu'il appelle au repentir et qu'il espère sensibles au souvenir de la grâce (Avertissement, n. p.). La consolation se présente comme une courroie d'affects, opérante pourvu que Dieu même y dispose les bénéficiaires. Sous sa plume, la consolation est un don. Elle est moins l'initiative d'un homme compatissant que l'effet d'une grâce, la marque d'une bénédiction divine qui en détermine l'efficace.

Cette Prière est l'occasion pour Beringhen de qualifier la consolation, qu'il tient pour semblable à une méditation du cœur. Elle est fondamentalement le lieu d'un discours militant qui en appelant Dieu à prendre pitié des apostats et à affermir les justes (et plus largement à soutenir les affligés), prie pour la destitution des "méchans " (persécuteurs et renégats endurcis), plaçant le discours de consolation directement sous l'égide divine, dans une perspective triomphale.

L'auteur adjoint une prière d'intercession (Article d'intercession, dans mes prières, depuis la révolte de ma femme) en faveur de son épouse qui a abjuré et des protestants qui ont apostasié (pièce sans date mais concomitante de la conversion au catholicisme de son épouse dont témoigne la lettre XII du 28 décembre 1686). La confidence ajoute à l'intimité du dispositif consolatoire, l'auteur exposant les faiblesses de ses plus proches parents, à l'instar de celles qui affectent une partie des réformés de France.

L'index (Indice des lettres), qui précise la date et le lieu de rédaction, l'identité d'une partie des destinataires, offre un bon aperçu de la composition de l'ouvrage et de la matière: les lettres d'une longueur variant de 3 à 40 pages s'adressent en grande majorité à des protestants, insoumis ou convertis («nouveau reüni» à la religion du roi), membres de la famille et proches parents ${ }^{28}$, connaissances ou amis, tout en incluant cinq missives ${ }^{29}$ adressées à des catholiques (amis ou polémistes). La plupart des lettres sont adossées à une péricope biblique que l'index ne reporte pas. Enfin, l'index renferme des missives sans destinataire précis qui forment autant de lettres de consolation types ${ }^{30}$.

On ne peut déterminer les critères de la sélection opérée par Beringhen dans le choix de ses lettres. En revanche, on peut appréhender les sujets de consolation: emprisonnement, exil, épreuve de la persécution et de l'apostasie, assignation, réclusion dans un couvent, éloignement et complainte des familles restées en France ${ }^{31}$, mort d'un proche ${ }^{32}$. Le recueil se partage entre lettres aux prisonniers, aux convertis ${ }^{33}$, aux veufs et veuves. Près de la moitié des lettres se concentrent sur les années 1686 et 1687, période d'intense activité législative et répressive ; période aussi de résistance active de l'auteur et d'abjuration de son épouse. Plusieurs missives, plus exhortatives que consolatoires et davantage polémiques (notamment adressées aux catholiques romains), se distinguent par leur tonalité controversiste.

30 Ces lettres écrites sur près de quinze ans, entre le 25 février 1686 et le 3 juillet 1700, le sont sans logique immédiatement perceptible ni argument particulier pouvant éclairer 
ces bornes chronologiques. Quatre ans se sont écoulés entre la rédaction de l'ultime lettre et la parution de l'ouvrage : est-ce le temps qu'il a fallu à l'auteur pour parvenir à l'éditer? Est-ce lié à sa libération, à la réactivation de l'oppression en France, à une conjoncture favorable au Refuge, à l'opportunité d'un financement, à l'activation des secours des exilés en faveur des réformés du royaume, à la considération tardive de l'utilité publique des lettres? Rien de tout cela, semble-t-il. Comme on le sait, l'auteur prétexte en premier lieu la prudence :

Ceux qui liront cet ouvrage [...] croiront vrai-semblablement que je pouvais me dispenser de les faire imprimer [...]. Mais j'espère des personnes équitables qu'elles se persuaderont aisément, que si j'ay eü des raisons d'en différer l'édition jusqu'à présent, j'en ai d'autres de ne la retarder pas plus long-temps. Comme il est malaisé qu'on n'y reconnoisse pas les personnes à qui j'écris, par divers caractères qui, pour ainsi dire, les montre au doigt, il auroit pu arriver divers inconvéniens d'une publication précipitée, qui auroit donné lieu de faire des affaires à ceux qu'on y auroit crû désignez, dans un temps où on en faisoit à tout le monde, sous des prétextes bien plus légers (Avertissement ${ }^{*}$ sq.).

Le propos n'est pas sans laisser poindre des différends au sein du Refuge quant aux choix tactiques et à la pertinence d'une publication tardive. Au reste, une certaine urgence paraît justifier le parti pris de l'édition: le maintien de la discrimination confessionnelle et les défaillances des réformés qui en ponctuent la réactivation lui paraissent être encore suffisamment fortes pour justifier la parution des lettres en 1704. Prétexte éditorial ou conjoncture décisive, l'impératif qui anime l'auteur échappe de prime abord à l'historien car, rappelons-le, 1704 n'est pas une date clé de la législation antiprotestante ${ }^{34}$, nous ne sommes pas au plus fort de la tempête révocatoire dont la dernière grande bourrasque remonte à la déclaration du 13 décembre 1698 venue relancer la persécution active des protestants du royaume et leur catholicisation. Ce calendrier suggère que le discours de consolation relève moins d'un souhait d'apaisement consécutif à une recrudescence de l'oppression (et donc des tourments) qu'à un propos d'alerte vis-à-vis de communautés qui s'enfoncent dans le nicodémisme ${ }^{35}$, se satisfaisant d'un compromis menaçant la survie même de la Réforme en France.

Le délai résulte du souci conjoint de ne certes pas exposer les destinataires à la répression du pouvoir français, mais aussi de la volonté de ne pas découvrir les convertis travaillant, à revers de leur abjuration de façade, à soutenir secrètement les insoumis :

De plus [en le publiant plus tôt], on auroit fait connoître aux ennemis de nôtre $S^{\text {te }}$ religion, la disposition d'un grand nombre de nouveaux reünis, qui n'aspiroient qu'à sortir de France pour mettre leur conscience en liberté, \& qui rendoient mille services à ceux qui persévéroient dans la confession de la Vérité; ce qui auroit donné sujet aux auteurs de nos misères, d'observer les uns de plus pres, \& de resserrer plus étroitement les autres [...], des considérations [...] qui engageoient a ne hazarder rien, \& par conséquent a différer une impression (Avertissement $*_{5 v^{\circ}}$ ).

La notion sous-jacente de service qui accompagne la présentation des lettres entrelacée à l'action des plus fervents, engagés dans le parti de l'insoumission spirituelle - rencontre un motif plus profond: lutter (une fois acquise la liberté de publier ces missives sans attenter à la vie de ses lecteurs) contre un statu quo funeste et une foi clandestine tacite :

Aujourdui toutes ces raisons ont cessé. Chacun a pris son parti en France. On n'expose plus personne en découvrant le commerce qu'on a eü avec quelques reünis [i.e. convertis], ou d'autres, il y a dix sept ou dix huit ans. On s'est accoutumé enfin 
au Conseil de France, a voir des gens qui ne peuvent oublier la Vérité qu'ils ont apprise dès leur enfance ; \& qui, s'ils ont perdu la liberté \& le courage d'en faire profession par des exercices publics, ne laissent pas de cultiver secretement la connoissance, \& d'en garder le dépost dans le fond du cœur. On [i.e. l'autorité politique] n'ignore pas même qu'on leur envoye de tous côtez des livres de piété qui les encouragent \& les consolent; \& que toutes les précautions de ceux qui les veulent priver de ce secours ne peuvent empescher qu'ils ne les reçoivent. Je n'ai plus aussi de considérations particulières qui me retiennent, \& par conséquent, je me trouve dans la pleine liberté de mettre ces Lettres en lumiere; comme il y a longtemps que j'en avois le désir (Avertissement, n. p.).

Ces deux extraits sont d'intérêt : l'auteur - qui, contre toute attente, donne à entendre la complicité des convertis, certes apostats mais pour une part sujets actifs du contournement de l'interdit religieux et acteurs engagés de la lutte réformée considère qu'en 1704 les autorités catholiques se sont accoutumées à la fidélité huguenote, en somme qu'il y a prescription à garder intimement sa foi. Beringhen, qui écrit au creux de la vague législative (la persécution ne connaît pas d'intensité comparable avant le nouvel élan oppressif du printemps $1724^{36}$ ), divulgue le motif de son entreprise, en même temps que le profil de ses nouveaux destinataires, et la raison de ce discours de consolation, qui entend clairement lutter contre un protestantisme résigné, persistant mais dissimulé : l'enjeu est celui de la visibilité d'une communauté dont les contours s'estompent au gré d'une piété clandestine. Le discours de consolation vise en soutenant les affligés et en reprenant les timorés à une audace de foi : une revendication publique de la croyance, une fidélité manifeste et notoire - un trait qui pose la question de la consolation en tant que discours sur la perte, s'élaborant face à ce qui menace de disparaître, sinon face à ce qui a déjà disparu. Demeure un fossé entre des lettres écrites au plus fort des tourments et une situation insatisfaisante mais apaisée: on s'interroge sur l'adéquation et la pertinence du propos, du fait de la nécessaire différence d'intensité entre le moment de rédaction des lettres et celui de leur diffusion. Ce qui laisse supposer que le discours de consolation n'est pas fonction d'une échelle de gravité (puisqu'il demeure valable quelle que soit l'intensité) mais fonction d'un acte, en l'occurrence d'un acte d'abandon: la consolation est une façon de prévenir un acte de résignation (à la souffrance), de renoncement (face à l'épreuve). À ce titre, la pertinence de ce discours de consolation repose, à vingt ans d'intervalle, moins sur la parenté (ou la permanence) des défis que sur l'expérience d'une ténacité, qui doit faire évaluer la nécessité du combat au bout duquel réside la liberté - telle que le confesseur/consolateur l'a lui-même arrachée.

Quoi qu'il en soit, ces missives se veulent l'expression et le témoignage d'un fidèle. Elles s'annoncent comme autant de réflexions visant à fortifier. Beringhen précise leur raison d'être :

Ce que je cherche en les publiant, c'est de renouveller le sentiment des graces que Dieu m'a faites, quand il a permis, qu'au milieu de mes adversitez, je me consolasse par ces méditations ; \& que roulant d'exil en exil, \& de prison en prison, je fusse capable \& de supporter ces épreuves, \& de travailler à donner du courage aux autres (Avertissement, n. p.).

Loin de toute idée de passivité, la confidence pose le discours de consolation comme un ressaisissement. Réversible, la dynamique consiste à consoler en édifiant (et de même par l'édification à réconforter) ceux qui ont trébuché, en particulier ceux qui, «après être tombé[s)", se repentent de leur abjuration, mais aussi de soutenir la détermination des persévérants restés dans le royaume : 
Si quelqu'un fait le même usage que moi de ces Lettres, \& qu'elles réveillent en lui le souvenir des graces qu'il a recuës de Dieu, ou en le fortifiant par de semblables pensées dans la confession de la Vérité, ou en le rappellant à le glorifier par la repentance après être tombé, ce sera un fruit de ce petit travail [...] \& si quelqu'un de ceux qui sont encore en France, en reçoit de l'édification \& en profite; ou pour s'affermir dans la Vérité, ou pour y revenir, je serai au comble de mes souhaits (Avertissement, n. p.).

Alors comment comprendre la prétention de l'auteur à n'écrire que pour les « cœurs capables de l'amour de Dieu »? Est-ce à dire que le discours de consolation serait conditionnel, fonction d'une attitude? On touche là à un paradoxe qui veut que la consolation s'adresse à un défaillant (et vient soutenir ou résorber une faiblesse), tout en exigeant une intégrité minimale (ici la repentance, la contrition). Il y a là une tension que l'on retrouve dans tous les discours de consolation huguenots, et en particulier dans la littérature apologétique post-révocatoire : un discours qui s'affiche contingent et qui pourtant se doit de n'exclure personne ni d'être en tout point catégorique s'il veut atteindre le plus grand nombre et espérer ramener les timides.

L'édition en 1704 ne doit pas faire oublier l'élaboration première qui maintient ce discours de consolation à la croisée du témoignage et de la méditation personnelle que l'auteur décide de livrer au public, à l'articulation de la confession et de l'exhortation (Article d'intercession). L'intentionnalité de l'auteur, qui revendique avoir écrit pour édifier (dédicace *3), est réaffirmée en divers textes du recueil. La consolation y est appréhendée comme "un moyen» d'édification, un "usage». Il s'agit, explique Beringhen, de fortifier par la pensée (Avertissement), et c'est là l'une des possibles définitions de la consolation.

Cette consolation recouvre aussi le souhait d'un retentissement et d'un bénéfice publics. L'auteur retrace la trajectoire d'un discours qui émane de la sphère privée (correspondances aux proches) pour ensuite gagner la sphère publique (à savoir la communauté réformée exposée aux interdits religieux et travaillée, sinon par le reniement, du moins par l'hypocrisie et la tentation du renoncement ${ }^{37}$ ). Mais que penser ou comprendre de la généralisation d'un discours intime qui, de privé devient public, d'individuel et personnalisé (adressé à une personne) s'adresse désormais à une communauté ? Cela pose la question de la codification du discours. Ce choix éclaire peut-être une autre facette du discours de consolation qui est une façon d'être partie prenante de l'épreuve, d'établir une proximité, de se montrer soi-même engagé, ici en intégrant au recueil les lettres confidentielles écrites à sa propre épouse. L'auteur se défend dans l'Avertissement de toute impudicité, d'une offense délibérée : partager les vicissitudes liées à l'apostasie de sa femme relève d'une parenté afflictive. N'est-ce pas une façon de se joindre aux destinataires à travers l'exposition de ses tourments? Revendiquant le «caractère de piété » de sa correspondance, il confère au discours de consolation une tonalité dévotionnelle. En étant de l'ordre de l'expérience (du partage d'une épreuve intime et du service), la consolation est encore de l'ordre de l'exercice de la foi. Elle se trouve d'ailleurs associée, dans l'un des extraits précédents, à un " petit travail ». Dans ce recueil qui s'apparente à un manuel de consolation, l'auteur la pense telle un acte de piété qui, face à la diversité des figures et des situations, doit favoriser la méditation et permettre l'apaisement des « autres dans leurs différentes afflictions » (Prière, n. p.). consolatoire. Discours de persévérance et d'espérance devant l'infortune, il est une 
façon d'énoncer une lutte solidaire dont il établit une filiation fondée sur l'exemple et le partage des tribulations. À la fois discours de célébration (de l'endurance, de la fermeté, de la fidélité et, plus fondamentalement, de la miséricorde divine) et d'alerte (contre l'inconstance religieuse et la damnation qui menace les impénitents), il se présente aussi comme un discours d'indignation qui sert une protestation de foi, tant contre l'épouvante des violences catholiques que contre la dissimulation de la foi réformée, face auxquelles Beringhen clame sa «mortelle aversion pour le déguisement " des cœurs ${ }^{38}$. Il permet de ménager compréhension (considération des peines et des faiblesses) et récrimination, ainsi d'articuler un discours volontiers charitable et néanmoins prescriptif. Sans rompre avec le propos compassionnel, le discours de consolation relève d'une forme de militance active dans laquelle l'adversité devient le ressort d'une lutte offensive exhortant à ne pas perdre la foi sinon à la défendre publiquement ${ }^{39}$ : «Il n'est pas d'un véritable chrétien de dissimuler sa créance ni de vivre sans exercice de religion ${ }^{40} »$.

Revenons, pour conclure, à la figure du consolateur/confesseur pour souligner que, conformément à l'économie symbolique huguenote, celui-ci n'est jamais chez Beringhen que l'instrument de Dieu, consolateur suprême. La constance procédant de l'unique dilection de Dieu, la consolation relève moins de la compassion humaine que de l'action divine : dans ce schéma, le confesseur/consolateur n'est pas le produit de sa propre force, mais l'instrument de Dieu qui lui donne le courage d'endurer l'injustice et de servir sa cause en soutenant les siens. Ceci pour dire, qu'ici du moins, le discours de consolation, qui se veut édifiant, est d'abord et avant tout un discours sur Dieu, supposant un état de confiance absolue et désignant en même temps une manifestation de sa toute-puissance. C'est en tout cas en "moindres instrumens » que l'auteur se perçoit et se revendique serviteur des éprouvés (Avertissement, n. p.).

La consolation des huguenots débute dans leur propre lecture des Écritures, qui cherche à comprendre le sens d'un temps d'épreuve au prisme d'une histoire biblique réconfortante. Elle ne consiste pas à faire disparaître les souffrances, mais à valoriser l'épreuve spirituelle, sans en rester à la lamentation qui, dans cette perspective, serait comme un déni de la marque élective dont gage paradoxalement l'oppression religieuse. La consolation réside dans la concession à la volonté divine. Ici, elle cherche moins à épargner qu'à galvaniser en sublimant l'objet même de la souffrance, à savoir la fidélité à Dieu. En ce registre apologétique, la consolation a pour particularité de viser à apaiser la douleur par le sujet même de l'épreuve. C'est dans l'affliction endurée pour la gloire de Dieu que réside la consolation ultime : objet de la souffrance, il est en même temps la raison de son soulagement. La consolation agit tel un manifeste de piété. Chez Beringhen, comme dans la littérature pastorale huguenote du temps, la consolation s'apparente à un énoncé de foi, plus encore, elle épouse les traits d'une «œuvre de la foy ${ }^{41}$ » qui se fixe, à l'appui de l'action même de Dieu, de ranimer la ferveur des affligés et d'entretenir leur patience. 


\section{ANNEXES}

\section{Lettre choisie (p. 461-465)}

Dans cette missive du printemps 1699, adressée à une protestante exilée au Refuge après avoir été soumise à diverses brimades, Beringhen livre une tierce définition de la consolation, qui consiste à donner à méditer sur la signification des vicissitudes, et à faire de la relecture scripturaire de l'épreuve un manifeste de dévotion dans lequel l'exercice de l'âme fidèle l'emporte sur l'agitation des entrailles. La consolation réside sinon est ce temps de relecture de la peine, ce dressage du corps, d'exercice de la foi. Chez ces huguenots pressés par la persécution des consciences, elle sert à clamer la promesse de salut et se présente comme un acte de foi. Une foi elle-même logée dans un acte de confiance qui consiste à s'en remettre à l'espoir d'une juste issue des souffrances endurées. La consolation est dans l'annonce d'une victoire sur la cruauté des persécuteurs et l'imminence du châtiment des oppresseurs que les huguenots pensent prochaines. Chez Beringhen, la consolation est un effet de Dieu. Elle est moins une façon de se résigner à la violence subie que de s'abandonner à la volonté divine.

La consolation réside dans ce procédé de transplantation, à la fois d'organes (du cœur vers l'âme) et de signifiances (de l'accablement à l'espérance). Elle est ce temps et ce moyen, ce processus de permutation de la douleur, de migration vers un autre que soi (de l'affligé à Dieu même). La lettre XLVIII illustre ce transfuge d'allégeances, de la douleur vers la cause, et ce changement d'épicentre. In fine - et là est la consolation -, ni l'éprouvé ni les oppresseurs ni le sujet initial de la peine n'en sont les acteurs et l'objet ultimes. La consolation est cette déprise de soi, ce changement de paradigme. Ce procédé est à l'œuvre dans la résolution de l'épouvantement dont s'agace Beringhen appelant à se dessaisir de l'effet pour en considérer la cause supérieure. En ce sens, la consolation est motrice. Rompant avec la fixité de la peine, elle est pareille à ces nouveaux cieux et cette nouvelle terre, dans la mesure où elle est une nouvelle appréhension de la douleur, un nouveau regard sur la frayeur qui la réoriente en direction d'une cause plus grande.

\section{Principes d'édition}

Nous conservons les graphies et italiques de l'originale, en particulier son usage irrégulier des accents (nous supprimons seulement son tréma sur eü ou obtenü, et ajoutons le tiret à " fût elle ", etc.); nous conservons également ponctuation et casse, à la seule exception des très nombreuses majuscules, que nous supprimons sauf dans le cas où elles désignent Dieu (« son Nom », « Celui qui ») ou Satan (« le Destructeur »), ainsi que « la Religion » ou encore « la Parole » (au sens de " parole de Dieu»). À la page 461, nous rétablissons " peuple», coupé par le passage à la ligne (l'originale a « le peu- » en fin de ligne, sans ple au début de la ligne suivante : « le peu-/qu'il s'est choisi »). 


\title{
XLVIII. LETTRE
}

\author{
A une Dame de ses amies, mise hors de \\ France, après y avoir souffert toutes \\ sortes d'injustices, \& de duretez \\ pendant bien des années, pour \\ la Religion. \\ Quand il aura connu le train que j'ay suivi ; \& \\ qu'il m'aura éprouvée, je sortiray comme l'or. Job 23.
}

Enfin Madame, après avoir lutté long-temps avec Dieu, pleuré, \& demandé grâce, vous en avez eu le dessus par sa miséricorde, \& obtenu l'issuë telle que vous l'aviez demandée. Je le bénis de tout mon cœur, de ce que vous ayant donné ; selon nos vœux, l'heritage de ceux qui craignent son Nom, il vous accorde aujourdui, une part si glorieuse entre ceux qu'il a honorez de ses délivrances. Puisez donc des fontaines de cette délivrance des eaux en joye, \& célébrez les exploits de l'Eternel; en vous éjouissant de la joye de sa nation, \& vous glorifiant avec le peu[ple] qu'il s'est choisi pour son plus précieux joyau. L'abondance des consolations dont vous allez être remplie, vous doit faire oublier les angoisses du passé. Il y a espoir pour tes derniers jours ; vous crient les Prophétes, tes enfans retourneront dans leurs quartiers ; \& ils seront comme autrefois. C'est là Mad. une promesse de salut \& de benediction : non seulement, vous la devez envisager, croire, \& saluer de loin, comme toutes les autres; mais vous devez encore l'embrasser par foy, comme [p. 462] si elle n'avoit été faite que pour vous. Vous avez été gardée immédiatement de Dieu dans la multiplicité de vos souffrances ; \& par la sapience qu'il a mise dans le secret de vôtre cœur, vous avez appris à vous résigner à sa volonté. Ce secours divin va se redoubler en vous, par les aides extérieures de la Parole, \& des sacremens ; dont vous avez été si long-temps privée. C'est donc aujourd'hui plus que jamais, qu'il faut que vôtre chair, \& les émotions de vos entrailles maternelles se taisent, en présence de Celui qui se dit le Dieu de toutes les familles de la terre. Il vient pour la juger ; que dis-je? Il se haste pour abréger l'affaire en justice, \& faire une consomption déterminée au milieu d'un peuple insensé qui a dépité son Nom. Encore un bien peu de temps, \& il fera venir à compte, le fruit de l'orgueil, \& de la cruauté du Destructeur. La terre sera mise en repos, quand il aura rompu le baston des méchans, \& la verge des dominateurs.

C'est Mad. en méditant ces célestes véritez, que vous vivrez en espérance, \& marcherez par foy, comme Dieu l'ordonne, dans cette nouvelle dispensation de sa Providence à vôtre égard. Il m'y fait vivre \& marcher aussi depuis bien des années : \& je puis dire, à la louange de la gloire de sa grâce en J. Christ, que c'a été sans varier ; \& sans qu'aucune considération de la terre ait été capable de m'ébranler en quoy que ce soit. Aussi ne me suis-je point épouventé, de ce qui a fait si souvent l'épouventement des autres ; \& de ce qui effraye encore aujourd'hui, je ne sçay quelle espece de réfugiez, qui ont toûjours les yeux tournez vers les persécuteurs de nos frères; comme si c'etoit uniquement du pouvoir de ces [p. 463] gens là, que deût venir le secours \& la délivrance. Au nom de Dieu, Mad. gardez-vous de cette contagion. Je sçay le scandale universel que cela cause dans tous les pays protestans, où Dieu nous a fait trouver des azyles. Il est vray que le nombre de ces réfugiez est très petit ; mais pour l'amour de J. Christ, ne vous laissez pas surprendre à leurs artifices, \& n'en fortifiez pas le party. Ce que le Seigneur Toutpuissant a opéré de grand, \& d'admirable en faveur de deux cent mille fugitifs, nous 
doit bien rassurer contre des frayeurs chimériques. On ne nous parle en secret que de la grandeur, \& de la prospérité d'une nation, \& de l'étenduë de ses forces : on voudroit nous convaincre que c'est d'elle ; \& non d'ailleurs, que nous pouvons espérer des radoucissemens. On la flatte même, \& on la caresse de la manière du monde la plus indigne, en la personne de ses Emissaires ; par tout où on les peut rencontrer. Mais fûtelle mille fois plus horrible \& plus terrible que les anciens Caldèens ; \& ses ESPIONS ENCORE PLUS AIGUS DE VEUE, QUE LES LOUPS DU SOIR, pour le dire avec le Prophéte Habacuc, graces à Dieu nous ne sommes plus comme autrefois sans dominateur \& sans appuy. Au contraire, il y a tout lieu d'espérer que le Rocher de nôtre délivrance ; qui a les yeux trop nets pour voir le mal, ne souffrira pas que le méchant dévore celui qui est plus juste que lui. Cet Arbitre souverain de tous les événemens du monde, a mille moyens pour nous procurer les temps de relasche \& de rafraichissement, \& l'An de salut qu'il promet au peuple de son Alliance. Si vous étant tenuë ferme à lui, dans la longueur de vos [p. 464] angoisses, vous éprouvez aujourd'hui si sensiblement, par vous-même, qu'il sçait délivrer de tentation ceux qui l'honorent, que ne devez-vous pas espérer pour tous ces captifs en France, qui gémissent, \& qui crient à lui jour \& nuit? Assurez-vous Mad. qu'il les vangera : que ce sera même bien-tôt ; * \& au moment qu'on y pensera le moins ; selon l'expression formelle \& précise de J. Christ, qui le promet dans son Evangile ${ }^{42}$.

C'est là Mad. a proprement parler, le fondement de l'espérance chrêtienne, \& ce qui peut faire le plus solide appuy de vôtre cœur, dans les ennuys que vous cause l'éloignement où vous vous trouvez, des personnes, a qui vôtre présence étoit si nécessaire. Il ne se peut, qu'à mesure que vous vous étes avancée vers le pays ou vous habitez présentement, vous n'ayez été remplie de quelcun de ces ravissemens, qui faisoient dire aux anciens fideles au retour de la Captivité ; Bénit soit l'Eternel qui n'a point permis que le torrent des eaux débordées soit passé sur mon ame! Puis donc Mad, que le Seigneur en vous transplantant comme par miracle, dans le lieu ou vous étes, semble y avoir créé pour vous de nouveaux cieux \& une nouvelle terre; selon la prediction d'Esaye, étudiez-vous a être trouvée de lui sans reproche, \& en paix à tous égards. Comme il se nomme expressément le Pere des orphelins, le Juge \& le Mary des veuves, croyez pour certain Mad. qu'il y a plus d'une bénédiction par devers lui, pour consoler toutes vos désolations. Je le prie de tout mon cœur, qu'il vous rende accomplie en toute bonne œuvre, faisant en vous [p. 465] ce qui est agréable devant lui par J. Christ. Je suis Mad. Votre. \&c.

Ce 2 Juin 1699.

\section{NOTES}

1. Ch. Bernat, «Protestantisme et fraternité : le langage de l'émotion. Les discours de consolation en temps d'épreuve (v. 1670 - v. 1720)», dans O. Christin, Y. Krumenacker dir., Les protestants à l'époque moderne. Une approche anthropologique, Rennes, Presses universitaires de Rennes, 2017.

2. Ch. Bernat, "Laodicée et la tiédeur sacrilège. Plaidoyer contre le scandale de la timidité spirituelle dans l'œuvre de Claude Brousson (1647-1698) », Études théologiques et religieuses, 90, 2015, p. 515-546 (en l'occurrence p. 536).

3. Mentionné dans de rares études historiques et notices biographiques du XIX ${ }^{\mathrm{e}}$ siècle, celui-ci n'a fait l'objet, à ma connaissance, d'aucune analyse de fond. Voir notamment infra n. 7.

4. Tandis qu'en régime catholique ce terme désigne aussi le prêtre qui a le pouvoir d'écouter les pécheurs et de leur donner l'absolution dans le cadre du sacrement de pénitence, il épouse dans 
la littérature apologétique huguenote la figure du réformé engagé au titre de sa foi et désigne le croyant qui endure l'épreuve au nom de la fidélité à sa croyance. Cette interprétation recoupe l'une des définitions donnée par Furetière, le confesseur étant ce «chrestien qui professe publiquement \& hautement la foy de Jésus-Christ, \& qui est prest de souffrir le martyre pour la soutenir " (dans A. Furetière, Dictionnaire universel, contenant generalement tous les mots en françoys, tant vieux que modernes, \& les termes de toutes les sciences et des arts, 3 t., La Haye, chez Arnout \& Reinier Leers, 1690). Attribué d'abord aux martyrs chrétiens, le qualificatif fut ensuite donné plus largement à ceux qui « après avoir été fort tourmentés par les tyrans, ont vécu \& sont mort en paix ». La précision n'est pas sans évoquer la dimension consolatoire de l'attitude du confesseur.

5. Cinquante lettres d'exhortation et de consolation sur les souffrances de ces derniers tems \& sur quelques autres sujets; écrites à diverses personnes par Monsr. D. V. B. pendant ses exils \& ses prisons en France, \& depuis que par ordre du Roi, il s'est retiré en Hollande, La Haye, chez Jean Kitto, 1704, lettres I: Théodore de Beringhen à Jean de Beringhen, Vézelay, 25 février 1686, p.1-10 et lettre XVI: Théodore de Beringhen à sa mère, Marie de Menours, château de Loches, 7 septembre 1687, p. 209-214. La notice de Jal indique que le père, épargné des moyens de rigueurs jusqu'en 1686, aurait abjuré le calvinisme sous l'effet de l'action du marquis de Vilette-Marsay, lieutenant des armées navales de Louis XIV, ce dont témoigneraient divers actes de catholicité consignés à Paris dans les registres de l'Église de Saint-Germain l'Auxerrois, tandis que les frères Haag mentionnent son renvoi de la cour dès novembre $1685 \mathrm{du}$ fait de sa fidélité à la Réforme (La Reynie l'aurait décrit «fort zélé dans sa religion »). Après son expulsion à Limoges à l'automne 1685 sur ordre de Louis XIV, Jean de Beringhen fut enfermé en décembre 1686 à la Bastille où il est enregistré parmi les prisonniers pour motif de religion, puis transféré au château d'Angoulême avant d'être expulsé du royaume avec son fils: A. Jal, Dictionnaire critique de biographie et d'histoire : errata et supplément pour tous les dictionnaires historiques d'après des documents authentiques inédits, Paris, Henri Plon, 1867, p. 201 sq. E. et É. Haag, La France protestante ou Vie des protestants français qui se sont fait un nom dans l'histoire depuis les premiers temps de la Réformation jusqu'à la reconnaissance du principe de la liberté des cultes par l'Assemblée nationale, Paris, Fischbacher, 1879, 1847, t. II, col. 337-343 (en particulier col. 340-341; la citation de La Reynie se trouve dans l'éd. de 1847 de La France protestante, t. II, p. 196).

6. Cet exemplaire annoté est conservé à la Bibliothèque du protestantisme français [désormais BPF] sous la cote $8^{\circ} 7877$ Réserve.

7. J. Chavannes, "Questions à propos d'un livre du Refuge ", Bulletin de la société de l'histoire du protestantisme français, 17, 1868, p. 42-48.

8. P. M. Conlon, Prélude au siècle des Lumières. Répertoire chronologique de 1680 à 1715, Genève, Droz, 1972, t. III, p. 328.

9. Voir E. et É. Haag, La France protestante, op. cit., t. II, col. 337-340.

10. Celle-ci aurait fait sa demande formelle de réunion au catholicisme auprès de Mme de Courtemer le 25 août 1687 et néanmoins les sources suggèrent sa conversion dès 1686 : E. et É. Haag, La France protestante, op. cit., t. II, col. 341, 343 (voir infra n. 17). La correspondance de Pierre Bayle (Correspondance, Oxford, Voltaire Foundation, 2001), qui entra au service de Jean de Beringhen en qualité de précepteur à partir du printemps 1675, fournit des indications complémentaires sur la famille: voir lettre 77 à Joseph Bayle, Rouen, 7 février 1675, t. II, p. 64-70; lettre 80 à Jean Bayle, 15 février 1675, p. 89-92 ; lettre 82 à Louis Tronchin, Paris, 22 mars 1675, p. 103 sq. ; lettre 83 à Jacques Basnage, Paris, 3 avril 1675, p. 105 et n. 2, p. 107 ; lettre 86 à Jean Bayle, Paris, 16 avril 1675, p. 130, n. 1. Voir de même les annotations de l'édition critique relative à la lettre 144 à Jacob Bayle, Sedan, 16 novembre 1677, t. II, p. 445, n. 12 à 14, tirées en partie de Tallemant des Réaux et de Saint-Simon (http://bayle-correspondance.univ-stetienne.fr).

11. La date de sa mort varie selon les sources. Le Dictionnaire généalogique, héraldique, chronologique et historique [...], Paris, Duchesne, 1757, t. I, p. 232 la date de septembre 1715. Les catalogues 
d'autorités des bibliothèques européennes - celui entre autres de la Bibliothèque nationale des Pays-Bas, tout comme le fichier d'autorité international virtuel (The virtual international authority file) - situent sa mort de manière concordante «na. [circa] 1703 ». Cette inexactitude complexifie l'interprétation de la parution du recueil, possiblement posthume donc.

12. Fr. Puaux, «Essai sur les négociations des réfugiés pour obtenir le rétablissement de la religion réformée au traité de Ryswick (octobre 1697)", Bulletin de la société de l'histoire du protestantisme français [dorénavant BSHPF] 16, 1867, p. 257-267 (p. 263).

13. Fr. Waddington, «Inventaire des manuscrits d'Antoine Court conservés à la Bibliothèque de Genève ", BSHPF 11, 1862, p. 80-104, en l'occurrence p. 94 (voir Papiers Court, ms. vol. R).

14. Voir É. Benoist, Histoire de l'Édit de Nantes, contenant les choses les plus remarquables qui se sont passées en France, avant \& après sa publication, à l'occasion de la diversité des religions, Et principalement les contraventions, inexecutions, chicanes, artifices, violences \& autres injustices, que les Réformez se plaignent d'y avoir souffertes, jusques à l'édit de révocation en octobre 1685, avec ce qui a suivi ce nouvel édit jusques à présent, 5 vol., Delft, Adrien Beman, 1693-1695, t. V, Livre xxIII, p. 872-873 (Benoist insiste sur l'intégrité et le zèle de Beringhen à l'égard de la religion réformée).

15. Avertissement, section non paginée [dorénavant n. p.]. Il n'a pas été possible d'identifier cet ouvrage. Aucune parution signée de sa main n'est recensée entre 1692 et 1703 dans le répertoire des publications du temps établi par Conlon.

16. Avertissement $*_{5} \mathrm{v}^{\circ}$ sq. On ne sait rien d'un éventuel retour au protestantisme. À l'appui des registres de la Chambre des comptes de Nantes et de la décision royale du 25 juillet 1686, les frères Haag signalent l'obtention par l'épouse et la fille de la jouissance provisoire des biens de l'ancien Conseiller du fait de leur conversion au catholicisme : E. et É. Haag, La France protestante, op. cit., t. II, col. 343.

17. Voir supra n. 11.

18. Le Thésaurus du Consortium of European Research Libraries le présente comme libraire éditeur en activité à s-Gravenhage [sic] (La Haye), Spuystraat, dès 1699 (et de même jusqu'en 1733).

19. L'auteur désigne "un cœur qui ne cherchoit qu'à se consoler en édifiant les autres». Voir Avertissement, section non paginée et l'Article d'intercession dans mes prières, depuis la révolte de ma femme ", n. p.

20. M. Fœssel, Le temps de la consolation, Paris, Seuil, 2015, $4^{\mathrm{e}}$ de couverture : "Consoler est une activité difficile qui implique de prendre la parole sur une souffrance que l'on ne partage pas, mais à laquelle on cherche à prendre part. Comment, sans la trahir, se frayer un chemin jusqu'à l'intimité de l'autre ? Quels mots employer qui ne suscitent pas le soupçon?».

21. Cette caractéristique consonne avec la définition qui présente la consolation comme «le produit d'une union des cœurs »: Cl. Martin-Ulrich, "Consoler à la Renaissance : discours privé ou acte public? ", dans E. Danblon dir., Usages et fonctions de la rhétorique. Regards interdisciplinaires sur la raison pratique, actes du Colloque international des 16-18 mai 2013 de l'Université Libre de Bruxelles, 2015 (en ligne).

22. L'ouvrage est présent dans le Catalogue des livres imprimez de la bibliothèque du roy, $3^{\mathrm{e}}$ partie : Théologie, Paris, 1742. Il compte actuellement un exemplaire disponible à la Bibliothèque royale de La Haye (cote KW $2217 \mathrm{E}$ 8), à la BPF ( $8^{\circ} 7877$ Réserve) et à la BnF (D2 - 4127), de même qu'une version numérique disponible en ligne (sur Google Livres).

23. La pagination est celle de l'exemplaire numérisé.

24. Avec ces lettres de consolation, écrit Beringhen, «je ne me suis pas proposé de donner au public un ouvrage qui le divertisse, mais qui l'instruise \& l'édifie» (op. cit., Avertissement).

25. Le titre de Hauts et puissants seigneurs (LL. HH. PP. : leurs hautes puissances) est celui ordinairement conféré à l'assemblée des États généraux des Provinces-Unies des Pays-Bas, qui étaient formés d'une délégation par province (délégations de Hollande, Frise, Zélande, Utrecht, Gueldre, Groningue et Overijssel). En 1704, le recueil paraît dans une période sans stathouder après la fin, en 1702, du stathoudérat de Guillaume III d'Orange-Nassau. 
26. Les Provinces-Unies y sont présentées comme «l'appuy de la Liberté publique, les Protecteurs des peuples opprimez, l'Azyle des personnes persécutées » (op., cit., dédicace *4).

27. Il y a une dimension conjoncturelle forte du propos consolatoire («l'occasion qui [...] les a dictées »). Quand bien même la matière peut s'avérer ré-employable, comme chez Beringhen, la composition est le fait d'un événement, sinon d'une rupture (une perte, un changement d'état, une épreuve).

28. Lettres I (« à Monsr. son Père », le 25 février 1686), XVI (« à Madame sa Mère », le 7 septembre 1687), XII, XIII, XVII à XXII, XXIV à XxIX («à Madame sa Femme ", entre le 21 décembre 1686 et le 16 février 1690), Xxxı («à Madame... sa belle-sœur», le 22 septembre 1691), xxxIv ("à un de ses Cousins Germains », le 6 novembre 1695), Xxxvı ("à son Frère Puis-né Nouveau Reüni », en Hollande, 13 janvier 1696) et XL (suite à «son Mariage avec une Dame Ancienne Catholique R. [omaine] », en Hollande, 14 juin 1696).

29. Lettres XxxIX ("à un Catholique Romain de ses Amis à la Cour de France», en Hollande, 13 mars 1696), XLII («à un Catholique Romain de ses Amis, en réponse », en Hollande, 22 février 1697), XLIV à XLVI (« à un Catholique Romain, en réponse à ce qu'il lui écrivoit », en Hollande, les 21 mars, 31 mai et 29 novembre 1697).

30. À l'exemple de la lettre XLIX du 18 septembre 1699 adressée «A Monsieur... Nouveau Reüni » (p. 465).

31. Lettres II-III (« à Mr. P. D. L. Hollandois », écrites depuis Beaulne, le 25 avril et le $1^{\mathrm{er}}$ mai 1686), IV, VII, IX, XV (« à Mr. de St. Hermines, prisonnier à la Bastille », écrites du même lieu, les 15 juillet, 12 août, 18 septembre 1686, 8 juin 1687), v («à une Dame de ses Amies réfugiée en Hollande », de la Bastille, le 20 juillet 1686), viII («à Madame L. D. D. L. F. retenue dans sa Maison, à Paris, par ordre du Roi », la Bastille, le 31 août 1686), x (« à Monsr. de Cagny, Prisonnier à la Bastille, du même lieu ", le 20 octobre 1686), XI et XIV (lettres anonymes à divers prisonniers de la Bastille, les 29 novembre 1686 et 20 avril 1687), xxx ("à une Demoiselle Réfugiée de ses amies », s. 1., le 23 février 1691), XXXIII ("à une Dame de ses Amies, renfermée dans le C.», en Hollande, le 22 novembre 1693), XXXVIII (« à une Demoiselle Réfugiée de ses Amies, pour le jour de sa Naissance, \& sur le grand Embarquement qui se faisoit à Dunkerque », s. 1., 6 mars 1696), XLVIII («à une Dame de ses Amies, mise hors de France ", s. 1., 2 juin 1699).

32. Lettres XXXII (« à Madame la C. D'Ol... sur la mort de Madame la P. D. T. sa Mère ", en Hollande, 3 mars 1693) ; XXXIv ("à un de ses Cousins Germains, sur la mort de son Frère, tué à l'assaut du Fort d'Orenge ", à Namur, s. 1., 6 novembre 1695), Xxxv (« à un des Seigneurs Régens du Pays, sur la mort d'une de [sic] Mesdemoiselles ses Filles», s. 1., 22 octobre 1695), XLI (« à une Demoiselle Réfugiée, sur la mort de sa fille unique, décédée en bas âge », s. 1., 4 décembre 1696), XLIII ("à une Dame de ses Amies en France, sur la mort de Mr. son mary », s. l., 28 février 1697), XLVII («à une jeune Dame de ses parentes, sur la mort de Mr. son Mary ", s. 1., 3 avril 1699), L (" à un homme de Considération de ses Amis, en Hollande, sur la mort subite de Madame sa Femme », s. l., 3 juillet 1700).

33. Lettres XXIII (« à Mr. D. C. Nouveau Reüni », d'Amsterdam, 16 mai 1688), XXXVI (« à son Frère Puis-né Nouveau Reüni », en Hollande, 13 janvier 1696), XXXVII (« à Monsr. D. Ch... Nouveau Réuni, gémissant en France », en Hollande, 2 février 1696), xLIX («à Monsieur... Nouveau Reüni, en réponse à la sienne ", en Hollande, 22 septembre 1699).

34. En 1703, l'essentiel des mesures prises à l'encontre des réformés est d'ordre fiscal et économique. En 1704, la législation fait état d'une déclaration du roi ( $1^{\mathrm{er}}$ mars) qui révoque les défenses faites à tous les sujets d'aller s'établir à Orange, et d'un Arrêt du conseil (2 août) concernant la régie et l'administration des biens de ceux de la R.P.R. [Religion prétendue réformée] sortis du royaume. 
35. Le terme désigne la duplicité confessionnelle qui consiste à dissimuler sa croyance en se montrant catholique de jour et protestant de nuit à l'instar de Nicodème qui n'approchait le Christ qu'en secret à la nuit tombée.

36. La déclaration du 14 mai 1724 est considérée comme l'une des inflexions les plus violentes de la chronologie révocatoire.

37. Op. cit., dédicace * 3 : «Je me suis déterminé à les publier, après les avoir long-temps gardées, parce que j'espère [...] [qu']elles pourroient encore servir à la consolation de ceux, à qui elles remettront dans la mémoire, les réflexions et les espérances qui soutiennent les enfans de Dieu dans leurs épreuves; \& parce qu[e] [...] j'ay crû qu'il se trouveroit un plus grand nombre de bonnes ames qui en pourroient profiter, si je les rendois publiques. »

38. Lettre XXVIII : lettre à Madame sa femme, en Hollande, le 29 janvier 1690, p. 305.

39. Voir, entre autres, la lettre Xxxvi écrite à son frère converti, en Hollande, le 13 janvier 1696, p. $372 \mathrm{sq}$.

40. Lettre XXVIII, p. 305.

41. Avertissement, n. p.

42. Note de l'édition, en bas de page, appelée au texte par l'astérisque : “* Luc. 18, " Soit Luc, 18,7 .

\section{AUTEUR \\ CHRYSTEL BERNAT}

Faculté de théologie protestante de Montpellier - Laboratoire d'études sur les monothéismes, UMR 8584 (CNRS - EPHE) 